\title{
Response to Immune Checkpoint Inhibition as Monotherapy or in Combination With Chemotherapy in Metastatic ROS1-Rearranged Lung Cancers
}

\author{
Noura J. Choudhury, MD, ${ }^{a}$ Jaime L. Schneider, MD, PhD, ${ }^{b}$ Tejas Patil, MD, ${ }^{c}$

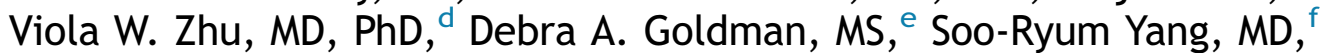 \\ Christina J. Falcon, MPH, ${ }^{\mathrm{g}}$ Andrew Do, BS, ${ }^{\mathrm{b}}$ Yunan Nie, MD, ${ }^{\mathrm{c}}$ \\ Andrew J. Plodkowski, MD, ${ }^{\mathrm{h}}$ Jamie E. Chaft, MD, ${ }^{\mathrm{a}, \mathrm{i}}$ Subba R. Digumarthy, $\mathrm{MD},{ }^{j}$ \\ Natasha Rekhtman, MD, PhD, ${ }^{f}$ Maria E. Arcila, MD, ${ }^{f}$ Alexia lasonos, PhD, ${ }^{e}$ \\ Sai-Hong I. Ou, MD, PhD, ${ }^{d}$ Jessica J. Lin, MD, ${ }^{b}$ Alexander Drilon, MD $^{a, i, *}$ \\ ${ }^{a}$ Department of Medicine, Memorial Sloan Kettering Cancer Center, New York, New York \\ ${ }^{b}$ Department of Medicine, Massachusetts General Hospital Cancer Center, Boston, Massachusetts \\ 'Department of Medicine, University of Colorado Anschutz Medical Center, Aurora, Colorado \\ ${ }^{d}$ Department of Medicine, University of California Irvine, Irvine, California \\ ${ }^{e}$ Department of Epidemiology and Biostatistics, Memorial Sloan Kettering Cancer Center, New York, New York \\ ${ }^{f}$ Department of Pathology, Memorial Sloan Kettering Cancer Center, New York, New York \\ ${ }^{\text {s}}$ Druckenmiller Center for Lung Cancer Research, Memorial Sloan Kettering Cancer Center, New York, New York \\ ${ }^{h}$ Department of Radiology, Memorial Sloan Kettering Cancer Center, New York, New York \\ 'Department of Medicine, Weill Cornell Medical College, New York, New York \\ ${ }^{j}$ Department of Radiology, Massachusetts General Hospital, Boston, Massachusetts
}

Received 6 May 2021; accepted 6 May 2021

Available online - 18 May 2021

\section{*Corresponding author.}

Drs. Choudhury, and Schneider contributed equally to this work.

Disclosure: Dr. Patil reports receiving honoraria from PRIME Oncology and Genentech/Roche. Dr. Zhu reports receiving honoraria from AstraZeneca, Blueprint, Roche-Foundation Medicine, Rochel Genentech, Takeda, and Xcovery and having stock from TP Therapeutics until May 2020. Dr. Goldman reports receiving stock from Johnson \& Johnson. Dr. Arcila reports receiving honoraria from Physicians' Education Resource, Biocartis, and Invivoscribe and having consulting or advisory role from AstraZeneca and Invivoscribe. Dr. Chaft reports having consulting/advisory role from AstraZeneca, Bristol-Myers Squibb, Genentech, Merck, Novartis, Regeneron, and Flame Biosciences. Dr. Digumarthy reports having consulting or advisory role from Merck, Pfizer, Bristol-Myers Squibb, Novartis, Roche, Polaris, Cascadian, AbbVie, Gradalis, Clinical Bay, and Zai Laboratories; receiving honorarium from Siemens Healthineers; and receiving research funding from Lunit, Inc. Dr. lasonos reports having consulting or advisory role from ICON Clinical Research, Intelligenica Inc., Journal of Clinical Oncology, and Mylan N.V. and intellectual property rights from Chapman and Hall. Dr. Lin reports having consulting/advisory role from Genentech, C4 Therapeutics, Blueprint Medicines, Nuvalent, Turning Point Therapeutics, and Elevation Oncology; receiving honorarium from Pfizer; receiving institutional research funding from Hengrui Therapeutics, Turning Point Therapeutics, Neon Therapeutics, Relay Therapeutics, Bayer, Elevation Oncology, Roche, and Novartis; and receiving CME funding from OncLive, MedStar Health, and Northwell Health. Dr. Drilon reports receiving honoraria from Medscape, OncLive, PeerVoice, Physicians' Education Resource, Targeted
Oncology, MORE Health, Research to Practice, Foundation Medicine, and PeerView; having consulting or advisory role from Ignyta, Loxo, TP Therapeutics, AstraZeneca, Pfizer, Blueprint Medicines, Roche, Helsinn Therapeutics, BeiGene, Hengrui Therapeutics, Exelixis, Bayer, Tyra Biosciences, Verastem, Takeda/Ariad/Millennium, BerGenBio, MORE Health, Eli Lilly, Verastem, AbbVie, 14ner/ Elevation Oncology, Remedica, Archer Biosciences, and Monopteros; receiving research funding from Foundation Medicine; having patents, royalties, and other intellectual property from Wolters Kluwer (Royalties for Pocket Oncology); and having other relationship from Merck, GlaxoSmithKline, Teva, Taiho Pharmaceutical, Pfizer, PharmaMar, and Puma Biotechnology. The remaining authors declare no conflict of interest.

Address for correspondence: Alexander Drilon, MD, Department of Medicine, Memorial Sloan Kettering Cancer Center, 545 East 73rd Street, 22nd floor, New York, NY 10021. E-mail: drilona@mskcc.org

Cite this article as: Choudhury NJ, Schneider JL, Patil T, et al. Response to Immune Checkpoint Inhibition as Monotherapy or in Combination With Chemotherapy in Metastatic ROS1-Rearranged Lung Cancers. JTO Clin Res Rep 2021;2:100187.

(c) 2021 The Authors. Published by Elsevier Inc. on behalf of the International Association for the Study of Lung Cancer. This is an open access article under the CC BY-NC-ND license (http:// creativecommons.org/licenses/by-nc-nd/4.0/).

ISSN: 2666-3643

https://doi.org/10.1016/j.jtocrr.2021.100187 


\section{ABSTRACT}

Introduction: ROS1 fusions are oncogenic drivers in 1\% to $3 \%$ of NSCLCs. The activity of immune checkpoint inhibitor (ICI) monotherapy or in combination with chemotherapy (chemotherapy with ICI [chemo-ICI]) in these tumors and their immunophenotype have not been systematically described.

Methods: In this multi-institutional retrospective study, tumor programmed death-ligand 1 (PD-L1) expression and tumor mutational burden (TMB) were evaluated in patients with ROS1-rearranged NSCLC. Time-to-treatment discontinuation (TTD) and objective response rate (ORR) (Response Evaluation Criteria in Solid Tumors [RECIST] version 1.1) were calculated for patients treated with ICI or chemo-ICI in the metastatic setting.

Results: A total of 184 patients were identified. Among 146 assessable cases, PD-L1 expression was less than $1 \%$ in 60 (41\%), $1 \%$ to $49 \%$ in $35(24 \%)$, and greater than or equal to $50 \%$ in 51 tumors (35\%). Of 100 (92\%) TMB-assessable tumors, 92 had less than 10 mutations per megabase. TMB was significantly lower for ROS1-rearranged tumors ( $\mathrm{n}=97)$ compared with tumors with EGFR $(\mathrm{n}=1250)$ or KRAS alterations $(\mathrm{n}=1653)$ and all other NSCLC tumors ( $\mathrm{n}=2753$ ) evaluated with Memorial Sloan KetteringIntegrated Mutation Profiling of Actionable Cancer Targets (median TMB $=2.6$ versus $3.5,7.0$, and 6.1 mutations per megabase, $p<0.001$ ). Among patients treated with ICI, median TTD was 2.1 months $(95 \%$ confidence interval [CI]: $1.0-4.2 \mathrm{mo} ; \mathrm{n}=28$ ) and ORR $13 \%$ (2 of 16 RECISTassessable; 95\% CI: 2\%-38\%). Among patients treated with chemo-ICI, median TTD was 10 months (95\% CI: 4.7$14.1 \mathrm{mo} ; \mathrm{n}=11$ ) and ORR 83\% (5 of 6 RECIST-assessable; 95\% CI: 36\%-100\%). There was no difference in PD-L1 expression $(p=0.91)$ or TMB $(p=0.83)$ between responders and nonresponders.

Conclusions: Most ROS1-rearranged NSCLCs have low PD-L1 expression and TMB. The activity of ICI in these tumors is modest. In contrast, chemo-ICI can achieve meaningful activity.

(c) 2021 The Authors. Published by Elsevier Inc. on behalf of the International Association for the Study of Lung Cancer. This is an open access article under the CC BY-NC-ND license (http://creativecommons.org/licenses/by-nc-nd/ $4.0 /$ ).

Keywords: Non-small cell lung cancer; ROS1 fusion; Immune checkpoint inhibitors; Tumor mutational burden; PDL1

\section{Introduction}

With multiple effective therapies approved in recent years, NSCLC provides a paradigm for precision medicine. Although targeted therapies against sensitizing
EGFR, ${ }^{1}$ BRAF $^{2}$ MET exon $14,{ }^{3} A L K,{ }^{4,5}{ }^{2} O S 1,{ }^{6,7} R^{2} T,{ }^{8}$ and $N T R K^{9}$ alterations are highly active in patients with advanced disease, therapeutic resistance is inevitable. The optimal sequence of later-line standard systemic options after disease progression on targeted therapies is unclear. Immune checkpoint inhibitors (ICIs) may be perceived as more attractive options for patients with programmed death-ligand 1 (PD-L1)-expressing tumors or tumor mutational burden (TMB)-high NSCLCs than combination regimens, such as platinum doublet or chemoimmunotherapy (chemotherapy with ICI [chemoICI]). Elucidating the activity of these nontargeted treatments in oncogene-driven tumors may help inform clinicians in optimally sequencing regimens.

ROS1 fusions are oncogenic drivers identified in at least 22 diverse adult and pediatric cancers, ${ }^{6}$ including NSCLC, gastrointestinal cancers, Spitzoid neoplasms, inflammatory myofibroblastic tumors, and gliomas. Given the annual global incidence of NSCLC, ROS1 fusions are most common in this tumor type despite comprising only $1 \%$ to $3 \%$ of cases. ${ }^{10,11}$ ROS1 tyrosine kinase inhibitors (TKIs) are the standard first-line treatment for metastatic ROS1-rearranged NSCLCs and are being investigated in other ROS1-rearranged cancers. ${ }^{12}$ In treatment-naive NSCLCs, crizotinib ${ }^{13-15}$ and entrectinib ${ }^{7}$ achieve high response rates (overall response rate $[\mathrm{ORR}]=69 \%-77 \%)$ and prolonged progression-free survival (PFS) (range: 19-21 mo). Next-generation TKIs, such as lorlatinib, ${ }^{16}$ repotrectinib, ${ }^{17}$ and taletrectinib, ${ }^{18,19}$ are active in TKI-treated NSCLCs. Although none are yet approved in this setting, lorlatinib use is supported by the National Comprehensive Cancer Network guidelines. ${ }^{20}$ Lastly, chemotherapy is active in ROS1-rearranged NSCLCs, with response rates of $45 \%$ to $60 \%$ and median PFS of 5 to 23 months ${ }^{6,21-23}$ for pemetrexed-inclusive regimens.

To date, very little is known on the immunophenotype and the activity of immunotherapy in ROS1-rearranged cancers. PD-L1 has only been reported in 10 patients across three reports, ${ }^{24-26}$ and TMB has not been characterized in a dedicated cohort of ROS1-rearranged NSCLCs. Only nine cases treated with ICI have been published, ${ }^{26,27}$ most of whom had primary progressive disease (PD); no cases treated with chemo-ICI have been reported. Lastly, it remains unclear whether high tumor PD-L1 expression ${ }^{28,29}$ or high TMB $^{30,31}$ should influence ICI therapy selection for these patients. To address these unmet needs, we launched a multiinstitutional, retrospective study of patients with ROS1-rearranged NSCLCs. The goal of this endeavor was to describe the incidence of ICI-predictive biomarkers and clinical outcomes of ICI as monotherapy or in combination with chemotherapy in patients with ROS1-fusion-positive NSCLC. 


\section{Materials and Methods}

\section{Study Population}

Patient eligibility criteria were as follows: (1) pathologically confirmed NSCLC; (2) ROS1 fusion positive; (3) any stage; and (4) treatment at one of the following four academic centers-Memorial Sloan Kettering Cancer Center, Massachusetts General Hospital, University of California Irvine, or University of Colorado. Patients had ROS1 fusions identified between August 1, 2011, and September 1, 2020. The study was approved by the institutional review board at each site, and patients provided consent. A retrospective review of medical records for demographic information, pathologic characteristics, medication administration, and radiologic response was performed.

\section{ROS1 Detection}

ROS1 fusions were detected by fluorescence in situ hybridization or next-generation sequencing (NGS) in a Clinical Laboratory Improvement Amendmentsapproved laboratory. NGS panels used to identify ROS1 fusion were as follows: Memorial Sloan KetteringIntegrated Mutation Profiling of Actionable Cancer Targets (MSK-IMPACT) $(\mathrm{n}=100)$; Massachusetts General Hospital Solid Fusion Assay $(\mathrm{n}=10)$; Foundation Medicine $(\mathrm{n}=5)$; Dana-Farber Cancer Institute OncoPanel $(\mathrm{n}=4)$; Guardant360 Cdx $(\mathrm{n}=3)$; Archer FusionPlex $(\mathrm{n}=3)$; and one each from OnkoSight, Tempus, Illumina TruSight, University of Washington Tumor Oncoplex, and Moffitt Cancer Center.

\section{Immunophenotyping}

PD-L1 and TMB were evaluated in ROS1-rearranged cancers of any stage with sufficient tissue for analysis as part of routine clinical care. TMB or PD-L1 evaluated at any point during a patient's disease course was accepted. Only one sample was used per patient. If patients had multiple samples with TMB or PD-L1 evaluated, results from samples with the highest tumor purity were selected. PD-L1 expression was scored by a pathologist as the percentage of tumor cells with membranous staining. PD-L1 antibodies used were Dako 22C3 (Agilent Technologies, Santa Clara, CA), E1L3N (Cell Signaling Technology, Danvers, MA), and Ventana SP142 and SP263 (Roche, Rotkreuz, Switzerland). Tumor PD-L1 expression was categorized consistent with prospective clinical trials ${ }^{32}$ as low $(<1 \%)$, intermediate $(1 \%-49 \%)$, and high $(\geq 50 \%)$. TMB was quantified by each respective NGS panel and defined as the number of nonsynonymous coding mutations per megabase (mut/Mb) of genome covered. TMB was quantified as low $(<10$ mut/Mb) and high $(\geq 10 \mathrm{mut} / \mathrm{Mb})$, consistent with the regulatory approval of pembrolizumab. ${ }^{31}$ Given the variability in TMB assessments across assays, for comparison of TMB between ROS1-fusion-positive tumors and ROS1-fusion wild-type tumors, the largest cohort of ROS1-rearranged NSCLCs with TMB data from a single NGS assay (MSK-IMPACT) $^{33}$ was chosen from the same time period.

To compare the immunophenotypic characteristics of ROS1 fusion-positive lung cancers to ROS1-wild-type lung cancers, two control populations were analyzed. For PD-L1 expression comparison, consecutive cases of ROS1 wild-type NSCLCs with tumor PD-L1 expression available from molecular profiling in the same time frame as the ROS1 fusion patients were identified using the NSCLC MSK-IMPACT cohort from the cBioPortal. ${ }^{34}$ The goal was to identify a cohort $2: 1$ in size relative to the ROS1 fusion-positive cohort. To compare TMB expression, all lung cancers with known ROS1 fusion status (fusionpositive or wild-type) by MSK-IMPACT were identified using cBioPortal.

\section{Therapy Activity}

The treatment cohort included any patient with ROS1 fusion-positive NSCLC who had received treatment with ICI monotherapy or chemo-ICI combination in the metastatic setting. Scanning intervals to evaluate treatment benefit were determined by the provider per standard of care and were not performed at consistent intervals. Best response was determined by Response Evaluation Criteria in Solid Tumors (RECIST) version 1.1 criteria for response-assessable patients with a baseline and one or more follow-up imaging studies. ORR was defined as the proportion of patients with a complete response or partial response (PR) as their best response to therapy; response confirmation by a second scan was not required.

\section{Statistics}

PD-L1 and TMB were categorized as noted previously. ORR on the basis of RECIST version 1.1 was estimated with 95\% exact confidence intervals (95\% CIs). Only patients with available RECIST assessments were included. The relationship between PD-L1 and TMB with ORR was evaluated with the Wilcoxon ranked sum test for continuous or ordinal values and Fisher's exact test for grouped values. We visually displayed the data with boxplots and bar charts overall and by treatment cohort (chemo-ICI and ICI); however, the subgroup sizes were too small for formal hypothesis testing. We also evaluated the relationship between maximum percent change in target lesions with PD-L1 and TMB using Spearman's correlation and visually displayed using scatter plots with the line of best fit. 


\begin{tabular}{|c|c|c|c|c|}
\hline Characteristics & $\begin{array}{l}\text { All ROS1-Fusion } \\
\mathrm{N}=184\end{array}$ & $\begin{array}{l}\text { Immunophenotypic } \\
\text { Cohort } \mathrm{n}=145\end{array}$ & $\begin{array}{l}\text { Treatment } \\
\text { Cohort } n=39\end{array}$ & $p$ Value \\
\hline Age, median (range), y & $51(20-89)$ & $56(31-87)$ & $56(20-89)$ & \\
\hline Sex & & & & 0.1 \\
\hline Male & $59(32)$ & $42(29)$ & $17(44)$ & \\
\hline Female & $125(68)$ & $103(71)$ & $22(56)$ & \\
\hline Race & & & & 0.4 \\
\hline Black & $16(9)$ & $11(8)$ & $5(13)$ & \\
\hline White & $116(63)$ & $92(63)$ & $24(62)$ & \\
\hline Asian & $39(21)$ & $33(23)$ & $6(15)$ & \\
\hline Not specified & $13(7)$ & $9(6)$ & $4(10)$ & \\
\hline Smoking status & & & & 0.002 \\
\hline Never smoker & $133(72)$ & $110(76)$ & $23(59)$ & \\
\hline$<10$ py & $31(17)$ & $17(12)$ & $14(36)$ & \\
\hline$\geq 10$ py & 19 (10) & $17(12)$ & $2(5)$ & \\
\hline Not specified & $1(1)$ & $1(1)$ & $0(0)$ & \\
\hline Stage, at diagnosis & & & & 0.6 \\
\hline I-III & $52(28)$ & $43(30)$ & $9(23)$ & \\
\hline IV & $132(72)$ & $102(70)$ & $30(77)$ & \\
\hline Tumor type & & & & 0.7 \\
\hline Adenocarcinoma & $179(97)$ & $141(97)$ & $38(97)$ & \\
\hline Squamous & $1(<1)$ & $1(1)$ & $0(0)$ & \\
\hline Adenosquamous & $3(2)$ & $2(1)$ & $1(3)$ & \\
\hline Not differentiated & $1(<1)$ & $1(1)$ & $0(0)$ & \\
\hline Brain metastases, at metastatic diagnosis & & & & 0.4 \\
\hline No & $125(68)$ & $99(68)$ & $26(67)$ & \\
\hline Yes & $45(25)$ & $33(23)$ & $12(31)$ & \\
\hline Not known & $14(8)$ & $13(9)$ & $1(3)$ & \\
\hline
\end{tabular}

Note: The immunophenotypic cohort consisted of 145 patients who had sufficient tissue for PD-L1 ( $\mathrm{n}=119)$ and/or TMB $(\mathrm{n}=77)$ testing. The treatment cohort consisted of patients who were treated with either immune checkpoint inhibitor monotherapy or chemoimmunotherapy.

PD-L1, programmed death-ligand 1; py, pack-year; TMB, tumor mutational burden.

Time-to-treatment discontinuation (TTD) for each treatment cohort was estimated using Kaplan-Meier methods with $95 \%$ log-log CIs and defined as the interval between the date of treatment initiation and the date of treatment discontinuation. Patients were censored at their last known clinical follow-up if they had continued treatment by the data lock on September 1, 2020. We compared TTD between the treatment cohorts using univariable Cox regression. The association between categorized PD-L1 and TMB with TTD was evaluated with the log-rank test, and univariable Cox regression was used for continuous PD-L1 and TMB. In those with available data, the associations between TMB and PD-L1 with ROS1 status were evaluated with the Wilcoxon ranked sum test for continuous/ordinal data and with Fisher's exact test for grouped data. Two-sided $p$ values less than 0.05 were considered statistically significant. Analyses were performed using Statistical Analysis System 9.4 TS1M6 (The SAS Institute, Cary, NC).

\section{Results}

\section{Patient Characteristics}

Between August 1, 2011, and September 1, 2020, a total of 184 patients with ROS1-rearranged NSCLC were identified (Memorial Sloan Kettering Cancer Center = 99, Massachusetts General Hospital $=43$, University of Colorado $=29$, University of California Irvine $=13$ ). Among all 184 patients, 52 patients (28\%) were diagnosed with stage I to III diseases and 132 (72\%) were diagnosed in the metastatic setting (Table 1 ). A total of 45 patients $(24 \%)$ had brain metastases at the time of diagnosis of metastatic disease. Most patients (89\%) were never or light former smokers $(<10$ pack-years) and had adenocarcinoma (97\%), consistent with the known clinical phenotype of ROS1-rearranged NSCLCs. ${ }^{11}$ The only statistically significant difference in baseline characteristics between patient cohorts was smoking status ( $p=0.002$ ). A higher proportion of patients in the treatment cohort had a light smoking history $(<10$ pack- 


\begin{tabular}{|c|c|c|c|c|}
\hline Characteristics & $\begin{array}{l}\text { All ROS1 Fusion Patients } \\
\mathrm{N}=184\end{array}$ & $\begin{array}{l}\text { Immunophenotypic } \\
\text { Cohort } n=145\end{array}$ & $\mathrm{ICI}$ Cohort $\mathrm{n}=28$ & $\begin{array}{l}\text { Chemo-ICI } \\
\text { Cohort } \mathrm{n}=11\end{array}$ \\
\hline \multicolumn{5}{|c|}{ Tumor PD-L1 expression } \\
\hline \# of patients & 146 & 119 & 17 & 10 \\
\hline Median (range) & $8(0-100)$ & $1(0-100)$ & $70(0-90)$ & $38(0-90)$ \\
\hline$<1 \%$ & $60(41)$ & $56(47)$ & $3(18)$ & $1(10)$ \\
\hline $1 \%-49 \%$ & $35(24)$ & $28(24)$ & $3(18)$ & $4(40)$ \\
\hline$\geq 50 \%$ & $51(35)$ & $35(29)$ & $11(65)$ & $5(50)$ \\
\hline \multicolumn{5}{|l|}{ TMB (mut/Mb) } \\
\hline \# of patients & 100 & 77 & 16 & 7 \\
\hline Median (range) & $3(0-20)$ & $3(0-20)$ & $3(1-20)$ & $2(1-5)$ \\
\hline$<5$ & $68(68)$ & $51(66)$ & $12(75)$ & $5(71)$ \\
\hline $5-9$ & $24(24)$ & $20(26)$ & $2(13)$ & $2(29)$ \\
\hline$\geq 10$ & $8(8)$ & $6(8)$ & $2(13)$ & $0(0)$ \\
\hline
\end{tabular}

Note: \# of patients refers to the number of patients in which PD-L1 or TMB, respectively, was available.

Chemo-ICI, chemotherapy with immune checkpoint inhibitor; ICl, immune checkpoint inhibitor; mut/mb, mutations per megabase; PD-L1, programmed deathligand 1; TMB, tumor mutational burden.

years) compared with patients in the full ROS1 cohort and the immunophenotypic cohort (36\% versus $17 \%$ and 12\%). ROS1 fusion was detected by NGS or fluorescence in situ hybridization in 129 patients $(70 \%)$ and 30 patients $(16 \%)$, respectively. CD74 was the most often identified fusion partner in 72 patients (39\%).

\section{Immunophenotype}

PD-L1 expression data were available for 146 patients with ROS1-rearranged NSCLCs $(\mathrm{n}=27$ treatment cohort, $\mathrm{n}=119$ immunophenotypic cohort). Tumor PDL1 expression was low $(<1 \%)$, intermediate $(1 \%-49 \%)$, and high $(\geq 50 \%)$ in $60(41 \%), 35(24 \%)$, and 51 patients (35\%), respectively (Table 2). PD-L1 expression was compared with a cohort of 291 ROS1 wild-type lung cancers that underwent consecutive PD-L1 testing during the study period. There was no significant difference in PD-L1 expression between the groups when examined as a continuous variable (median: $7.5 \%$ in ROS1 fusion versus $5.0 \%$ in ROS1 wild-type, $p=0.81$ ) or categorically (33 versus $40 \%, p>0.95$, Fig. $1 A$ ).

TMB data were available for 100 patients with ROS1rearranged NSCLCs $(n=23$ treatment cohort, $n=77$ immunophenotypic cohort). A total of 92 patients had tumors with less than $10 \mathrm{mut} / \mathrm{mb}$ (92\%), and eight patients (8\%) had greater than or equal to 10 mut/Mb. To avoid comparing TMBs quantified from different NGS panels, we restricted the comparative analysis to the cohort of patients who had undergone NGS and TMB determination by MSK-IMPACT, the most frequently used panel. The median TMB of ROS1 fusion-positive lung cancers (median $=2.6 \mathrm{mut} / \mathrm{Mb}, \mathrm{n}=97$ ) was significantly lower compared with tumors with EGFR (median $=3.5 \mathrm{mut} / \mathrm{Mb}, \mathrm{n}=1250$ ) or KRAS alterations (median $=7.0, \mathrm{n}=1653$ ) and compared with all other
ROS1 wild-type lung cancers (6.1 mut/Mb, $p<0.001$; Fig. $1 B$ and $C$ ). A breakdown of TMB by molecular subtypes of ROS1 wild-type lung cancers is illustrated in Supplementary Table 1. Among 69 patients who had both PD-L1 expression and TMB evaluated, only one patient had both high levels of TMB and PD-L1 expression (Fig. 1D).

\section{Activity of ICl monotherapy}

A total of 28 patients with advanced ROS1-rearranged NSCLCs were treated with ICI monotherapy (Supplementary Table 2): nivolumab $(\mathrm{n}=13)$, pembrolizumab $(n=12)$, investigational agent $(n=2)$, and atezolizumab $(\mathrm{n}=1)$. Time on therapy is illustrated in Figure $2 A$. The median TTD was 2.1 months $(95 \% \mathrm{CI}$ : $1.0-4.2, \mathrm{n}=28$; Fig. $2 B$ ). Reasons for treatment discontinuation were PD $(n=23)$, toxicity $(n=3)$, and planned discontinuation (elective switch to ROS1 TKI without evidence of progression, $n=1$ ); one patient continued ICI at the time of data censoring.

Of the 28 patients, 16 had RECIST reads performed retrospectively. Best response of complete response, PR, stable disease, and PD was observed in zero, two (13\%), four (25\%), and 10 patients (63\%), respectively (Supplementary Table 3). The ORR was 13\% (95\% CI: $2 \%-38 \%$, Fig. 2 C). Furthermore, $17 \%$ of patients (2 of 12) with baseline brain metastases had intracranial progression on ICI and $14 \%$ of patients ( 2 of 14 ) without baseline brain metastases had new intracranial metastases on ICI. Two patients did not have pretreatment or on-treatment central nervous system imaging performed.

The two patients treated with ICI therapy for more than 12 months both had low-volume disease (Fig. 2A). Patient 28 (TMB and PD-L1 unknown) was treated with 
atezolizumab in the fourth-line setting after progression on two TKIs and chemotherapy and received radiation to an unmeasurable left lung lesion during treatment; best response was evaluated clinically as stable disease. Patient 21 (TMB = $5.3 \mathrm{mut} / \mathrm{mb}$, PD-L1 0\%) received nivolumab as second-line therapy after chemotherapy and was on observation for nearly eight months after mild disease progression on nivolumab before requiring treatment with entrectinib.

\section{Activity of Chemoimmunotherapy}

A total of 11 patients were treated with chemo-ICI combination. Most patients (10 of 11) were treated with carboplatin, pemetrexed, and pembrolizumab (Supplementary Table 4). Time on therapy is found in Figure $2 D$. The median TTD was 10.0 months $(95 \% \mathrm{CI}$ : 4.7-14.1; Fig. 2E). Patients discontinued treatment for $\mathrm{PD}(\mathrm{n}=6)$ and planned discontinuation (treatment holiday and elective switch to ROS1 TKI, $\mathrm{n}=2$ ); three patients continued treatment at time of data censoring. One of two patients with baseline brain metastases had intracranial progression on chemo-ICI; no other patients developed new brain metastases on treatment.

Of the 11 patients, six had RECIST reads performed retrospectively. Five of six patients had a PR as the best response, resulting in ORR of $83 \%$ (95\% CI: 36\%-100\%, Fig. $2 F$ and Supplementary Table 3). By univariable Cox analyses, receiving chemo-ICI compared with ICI monotherapy was associated with a reduced risk of treatment discontinuation (hazard ratio $=0.37,95 \%$ CI: 0.16-0.82, $p=0.015$, Supplementary Table 5).

\section{Biomarker-Activity Correlations}

The relationships between tumor PD-L1 expression and TMB with response to ICI and chemo-ICI were evaluated. Patients were initially considered as
A
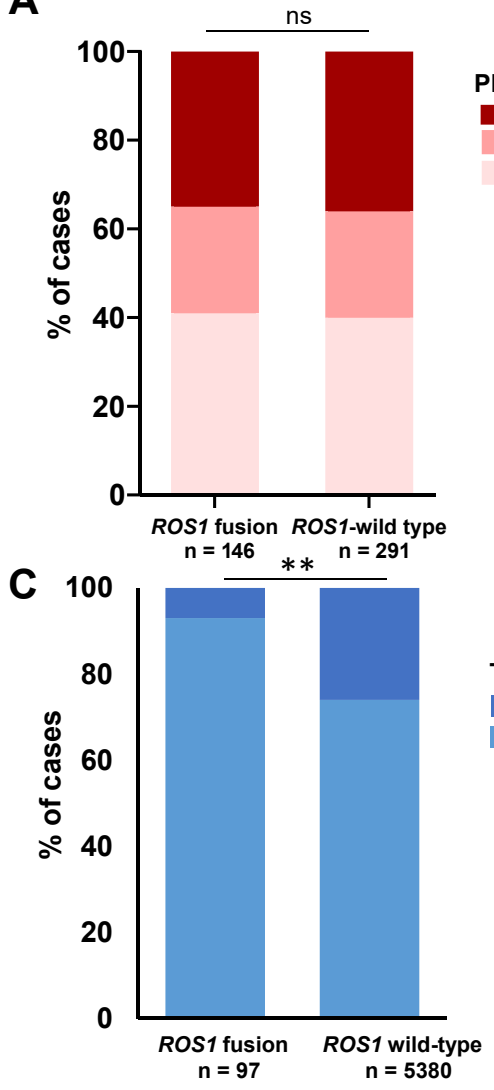

B
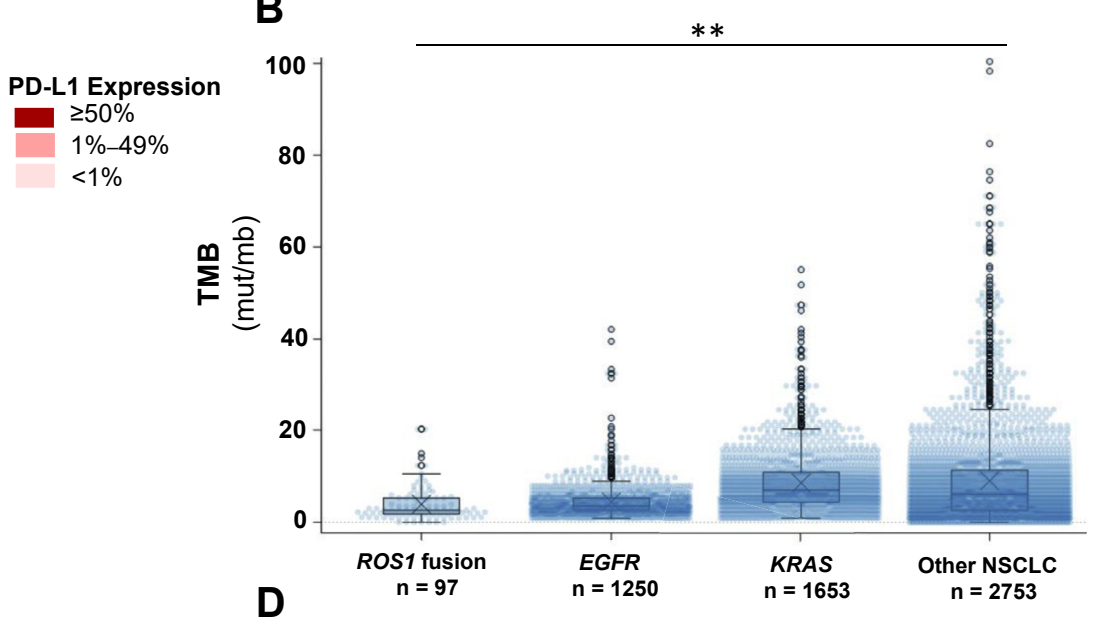

TMB $\geq 10$ mut/Mb $<10$ mut/Mb

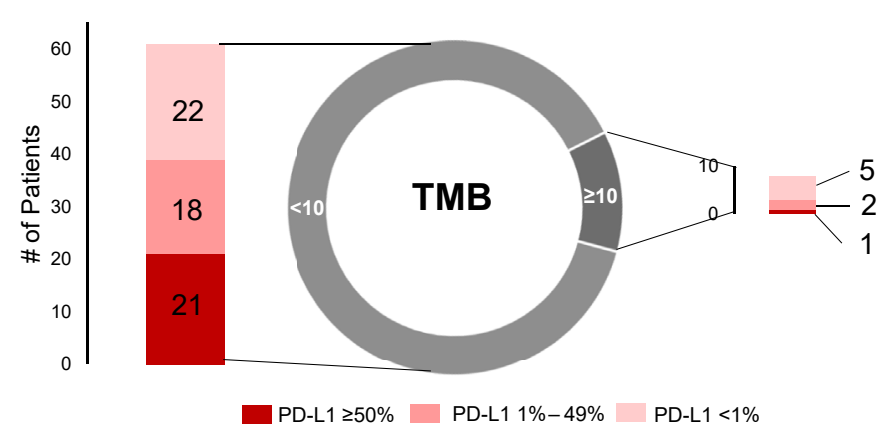

Figure 1. Immunophenotypic characteristics of ROS1-rearranged NSCLC. $(A)$ Comparison of categorical distribution of tumor PD-L1 expression for ROS1 fusion-positive and ROS1-wild-type NSCLC tumors $(p=0.9)$. (B) Comparison of TMB for tumors with ROS1 fusion, EGFR and KRAS alterations, and all other NSCLC tumors. Only tumors that underwent next-generation sequencing with MSK-IMPACT were included in this analysis. $(C)$ Comparison of categorical distribution of TMB between ROS1 fusion-positive and ROS1-wild-type tumors. Of 97 tumors (7\%) with ROS1 fusions, 7 had TMB greater than or equal to 10 mut/Mb compared with 1379 of 5380 tumors $(26 \%)$ without ROS1 fusions $(p<0.001)$. (D) Comparison of the overlap between TMB (any assay) and tumor PD-L1 expression for the 69 patients with both assessments available. ${ }^{* *}$ indicates $p<0.001$. MSKIMPACT, Memorial Sloan Kettering-Integrated Mutation Profiling of Actionable Cancer Targets; mut/Mb, mutation per megabase; PD-L1, programmed death-ligand 1; TMB, tumor mutational burden. 
A

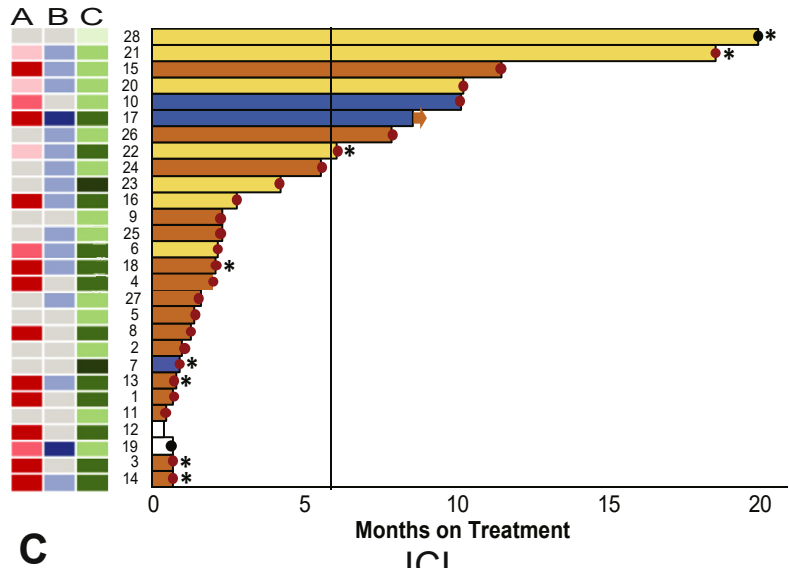

$\mathrm{ICl}$

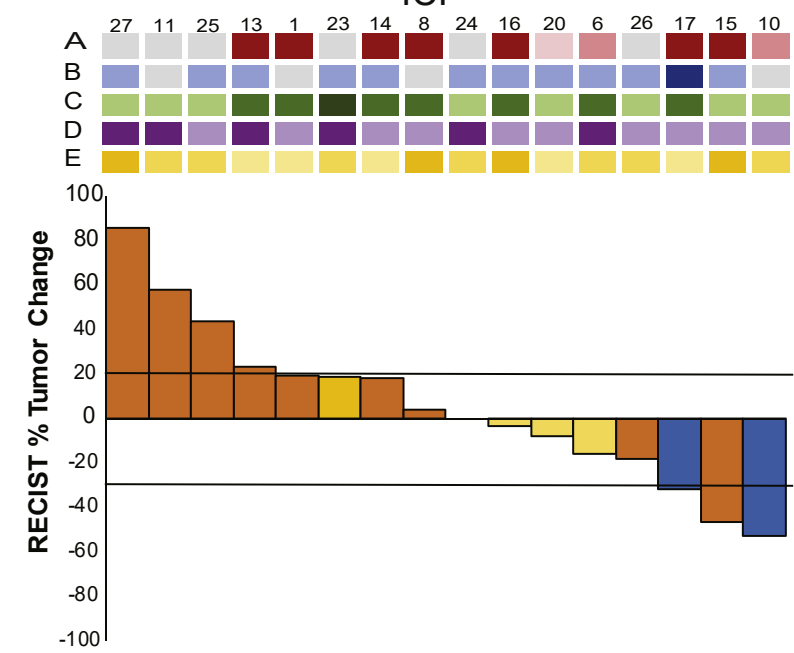

E

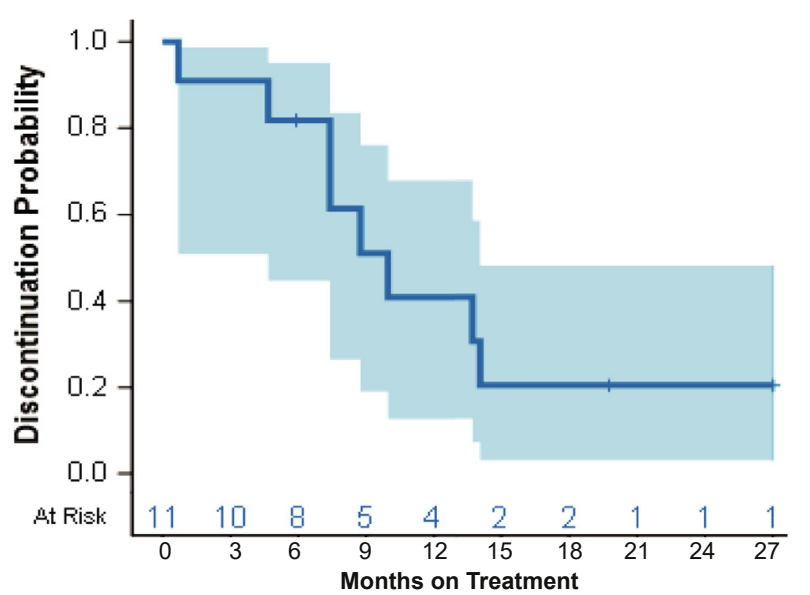

B

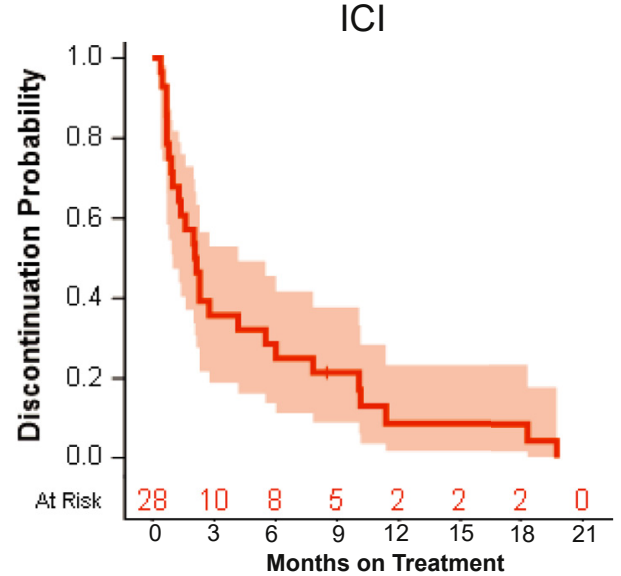

D
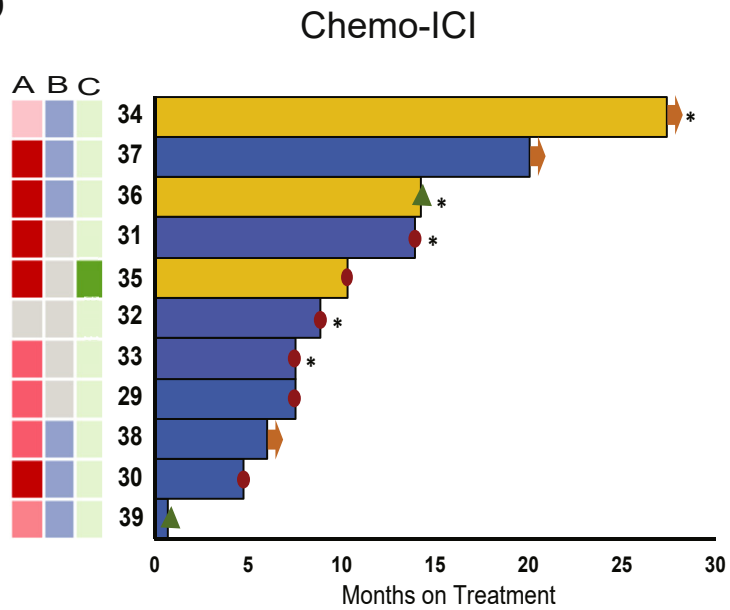

F

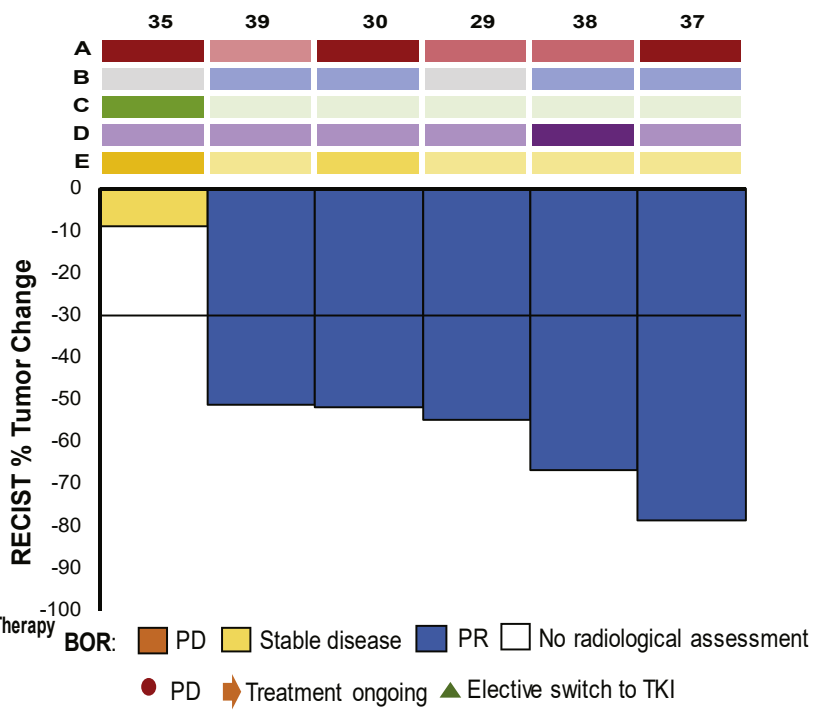

Figure 2. Clinical outcomes on $\mathrm{ICl}$ and chemo-ICl. (A) Swimmer's plot for 28 patients treated with $\mathrm{ICl}$. Asterisks indicate best response determined clinically. $(B)$ TTD for patients treated with $\mathrm{ICl}$ with $95 \%$ confidence intervals revealed. $(C)$ Waterfall plot of patients treated with ICI ( $n=16$ assessable for RECIST response). Note that patient 15 met RECIST criteria for PD for growth in nontarget lesions, although tumor shrinkage in target lesions was greater than 30\%. (D) Swimmer's plot for 11 patients treated with chemo-ICI. $(E)$ TTD for patients treated with chemo-ICI with $95 \%$ confidence intervals revealed. $(F)$ Waterfall plot of patients treated with chemo-ICI ( $n=6$ assessable for RECIST response); for patient 40, follow-up scan was 
A

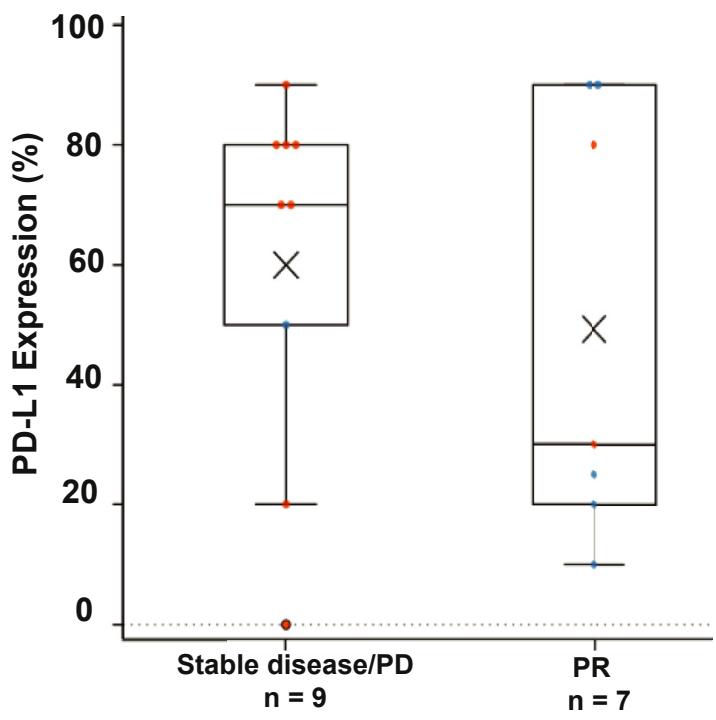

C

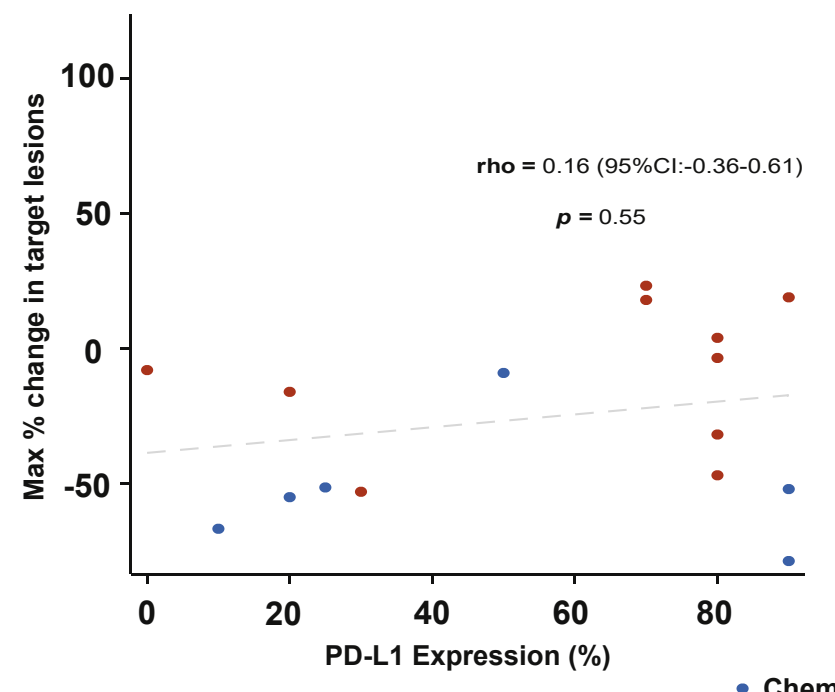

B

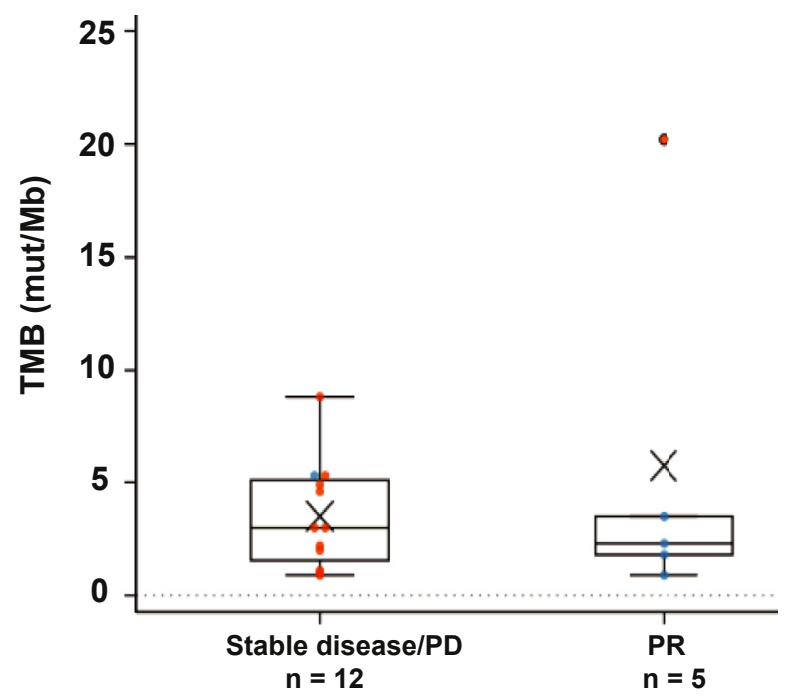

D

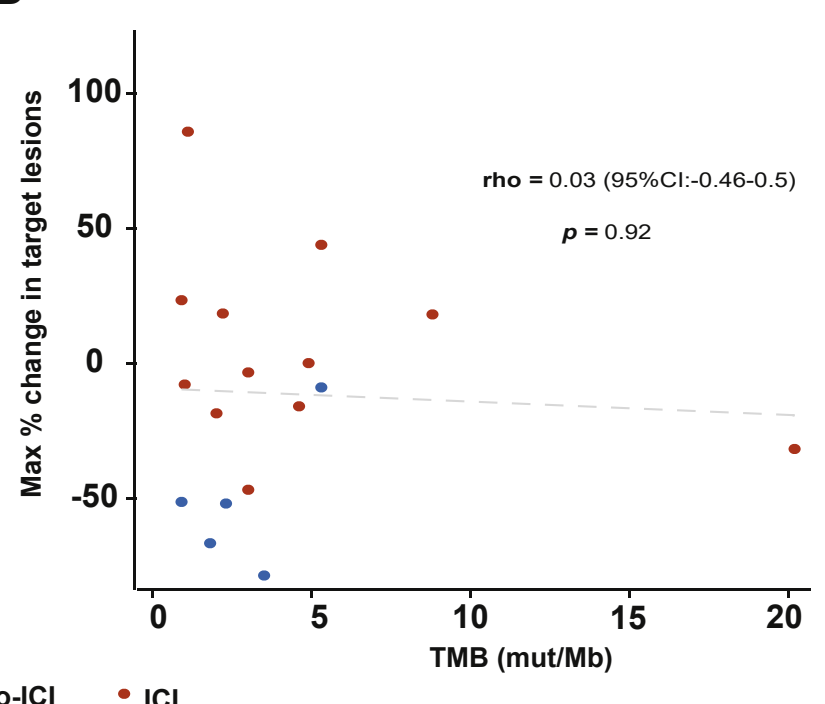

Figure 3. Impact of immunophenotype on ICI or chemo-ICI response. Only patients with RECIST evaluations available were included in this analysis. $(A)$ Comparison of PD-L1 expression between responders (best response PR) and nonresponders (best response stable disease and PD) to chemo-ICl or $\mathrm{ICl}$ (median $30 \%$ versus $70 \%, p=0.9$ ). (B) Comparison of TMB among responders and nonresponders with TMB available (median 2 versus 3 mut/Mb, $p=0.8$ ). (C) Correlation between PD-L1 expression and maximum change in sum of target lesions. $(D)$ Correlation between TMB and maximum change in sum of target lesions. Chemo-ICl, chemotherapy with immune checkpoint inhibitor; $\mathrm{Cl}$, confidence interval; ICl, immune checkpoint inhibitor; Max, maximum; mut/Mb, mutation per megabase; PD, progressive disease; PD-L1, programmed death-ligand 1; PR, partial response; TMB, tyrosine kinase inhibitor.

separate treatment cohorts; however, sample sizes were too small for formal hypothesis testing. When responders to chemo-ICI and ICI were combined, there was no significant difference between PD-L1 expression levels (median 30 versus $70 \%, p=0.91$,
Fig. 3A, Supplementary Fig. 1) or TMB (median 2 versus $3 \mathrm{mut} / \mathrm{mb}, p=0.83$, Fig. $3 B$ ) between responders (best response $\mathrm{PR}$ ) and nonresponders (stable disease/PD). There was also no correlation between maximum change in sum of target lesions

obtained shortly after patient transitioned to crizotinib. Chemo-ICl, chemotherapy with immune checkpoint inhibitor; ICl, immune checkpoint inhibitor; PD, progressive disease; PD-L1, programmed death-ligand 1; PR, partial response; RECIST, Response Evaluation Criteria in Solid Tumors; TKI, tyrosine kinase inhibitor; TMB, tyrosine kinase inhibitor; TTD, time to treatment discontinuation. Patient-level information corresponding to patient IDs can be found in the Supplement. 
with PD-L1 expression (rho $=0.16,95 \%$ CI: $-0.36-$ $0.61, p=0.55$, Fig. $3 C$ ) or TMB (rho $=0.03,95 \%$ CI: $-0.46-0.50, p=0.92$, Fig. $3 D$ ). Although we also evaluated the association between TTD and PD-L1 and TMB for each treatment cohort individually, sample sizes were again too small to obtain meaningful Kaplan Meier estimates. Nevertheless, in total, 11 patients with PD-L1 expression greater than or equal to $50 \%$ were treated with ICI, with a median TTD of 2.4 months (95\% CI: $0.7-11.4 \mathrm{mo}$ ). When the cohorts were combined, by univariate Cox analyses, neither TMB nor PD-L1 expression affected TTD (hazard ratio $=0.98, p=0.80$ and 0.27 , Supplementary Fig. 2 and Supplementary Table 5).

Other putative genomic modifiers of immunotherapy activity, such as alterations in STK11/LKB1, KEAP1, and PBRM1, ${ }^{35-37}$ were explored. Of 112 ROS1-rearranged lung cancers wherein information on these genes was available, inactivating mutations were observed in a minority of cases: 3 PBRM1, 1 KEAP1/PBRM1, 1 STK11/ PBRM1, and 1 KEAP1/STK11 (Supplementary Table 6). One patient with concurrent PBRM1/STK11 mutations received nivolumab (TTD 0.8 mo, RECIST radiological response not assessed), and a second patient with a PBRM1 Q1400* mutation received chemoimmunotherapy (TTD $4.7 \mathrm{mo}$, PR).

\section{Comparison With TKI Therapy}

Time on ICI monotherapy or chemo-ICI was qualitatively compared with time on ROS1 TKI therapy in patients who received both during their disease course. For this exploratory analysis, we focused on patients who remained on ICI treatment for at least six months $(n=8)$ and all 11 patients who received chemo-ICI. Patients were treated with crizotinib $(n=17)$, entrectinib $(n=9)$, lorlatinib $(\mathrm{n}=8)$, repotrectinib $(\mathrm{n}=6)$, cabozantinib $(\mathrm{n}=4)$, ceritinib $(\mathrm{n}=1)$, and alectinib $(\mathrm{n}=1)$. Swimmer's plot of time on ICI or chemo-ICI versus total time on one or more ROS1 TKIs is found in Figure $4 A$ and $B$. For patients treated with ICI, median cumulative time on all ROS1 TKIs exceeded median time on ICI (median 18.1 versus $2.1 \mathrm{mo}$ ); the same was true for patients treated with chemo-ICI (median 17.2 versus 8.7 mo, Fig. 4C, Supplementary Table 7). Patients in both groups spent their longest time on their first ROS1 TKI (median 10 mo for ICI cohort and 13.3 mo for chemo-ICI cohort).

\section{Discussion}

This multi-institutional study represents the largest series to date of ROS1-rearranged lung cancers that describes the immunophenotype and the activity of ICI and chemo-ICI in these tumors. Recognizing the limitations of this article and cross-trial comparisons, these data establish real-world historical benchmarks against which ongoing or future trials of systemic therapy for patients with ROS1 fusion-positive NSCLC can be evaluated. In patients with ROS1-rearranged lung cancers, late-phase trials randomizing patients to an immunotherapy-inclusive standard-of-care control arm will be challenging to execute.

PD-L1 expression was below 50\%, and TMB was less than $10 \mathrm{mut} / \mathrm{Mb}$ in most patients with ROS1-rearranged NSCLCs. The median TMB was significantly less that of other lung cancers, including EGFR-driven lung cancers, cancers that similarly arise in patients largely without a smoking history. Low TMB has similarly been reported in other oncogene-driven lung cancers. ${ }^{38-40}$ As expected, TMB was significantly higher in patients with KRAS alterations, which are more often found in patients with smoking histories. Given this immunophenotype, it is unsurprising that single-agent immunotherapy achieved a low response rate $(\mathrm{ORR}=13 \%)$ and short time on therapy (median TTD $=2$ mo). Furthermore, although the sample size is small, 11 patients with high PD-L1 tumor expression had a short median TTD of 2.4 months and most had primary disease progression. We further found no association between PD-L1 expression, TMB, and objective response or TTD.

Our findings of suboptimal response to ICIs among ROS1-rearranged lung cancers are consistent with the modest benefit from ICIs in other oncogene-driven lung cancers, such as those with sensitizing EGFR mutations. ${ }^{41-43}$ In one series, median PFS for EGFR-mutant NSCLC treated with ICI was 1.8 months. ${ }^{41}$ In the IMMUNOTARGET registry ${ }^{26}$ that evaluated the activity of single-agent immunotherapy in ALK, ROS1, and RET fusion-positive NSCLCs, primary PD was the most common outcome. Given these poor results, the National Comprehensive Cancer Network guidelines discourage later-line use of ICI in patients with EGFR or $A L K$ alterations. ${ }^{20}$ In a separate series, the response to singleagent immunotherapy in BRAF V600E-mutant, ${ }^{44}$ ERBB2-mutant, ${ }^{26}$ and MET exon 14-altered ${ }^{45}$ NSCLCs was $25 \%, 7 \%$, and $17 \%$, respectively, and the median PFS was $3.7,2.5$, and 1.9 months, respectively.

In contrast, acknowledging the limited sample size of this population, the activity of chemoimmunotherapy was more encouraging. All responseassessable cases had disease regression with most patients achieving a PR (ORR $=83 \%)$. The median TTD was 10 months. For reference, in KEYNOTE-189, which established combination chemoimmunotherapy as a standard of care for treatment-naive patients with metastatic NSCLC, the ORR was $48 \%$ and the median PFS was 9 months. ${ }^{46}$ Pending further confirmation, this suggests that combination chemoimmunotherapy might represent a preferred approach compared with 
A

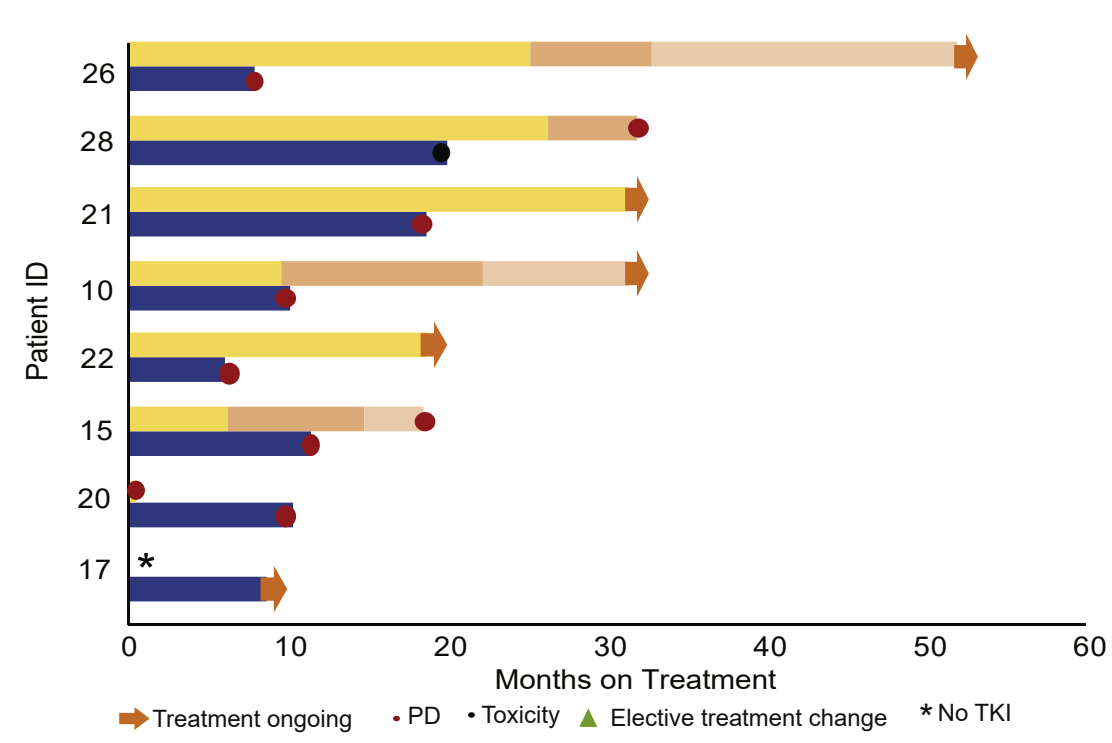

B

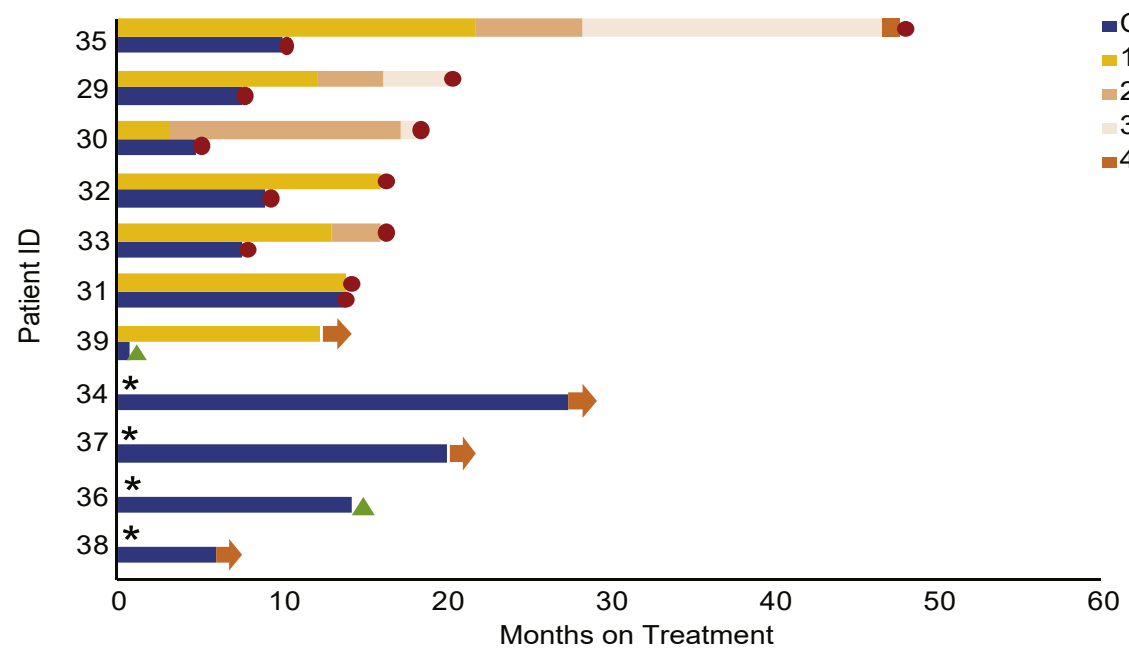

$-\mathrm{ICl}$

1st TKI

2nd TKI

3rd TKI
-Chemo-ICl

1st TKI

2nd TKI

3rd TKI

4th TKI

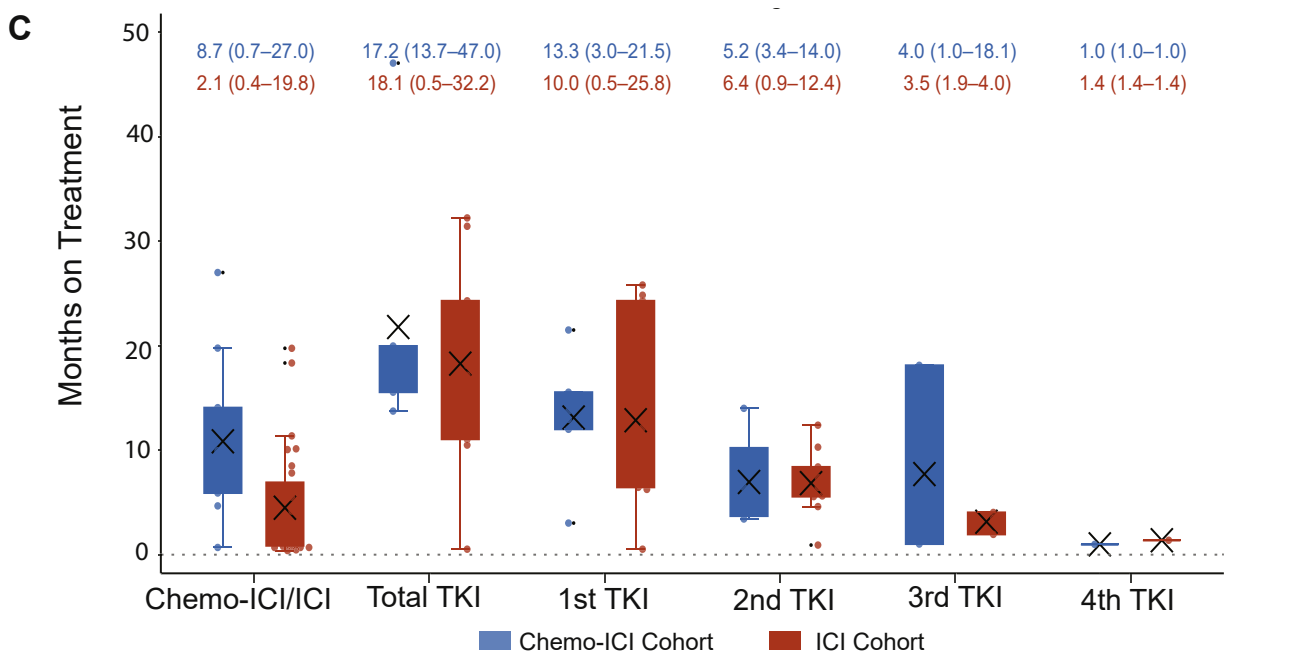

Figure 4. Time on $\mathrm{ICl}$ or chemo-ICl compared with ROS1 TKIs. (A) Swimmer's plot comparing time on ICI to ROS1 TKIs for patients who remained on $\mathrm{ICI}$ for at least six months $(\mathrm{n}=8)$. $(B)$ Swimmer's plot comparing time on chemo-ICI versus ROS1 TKIs for all patients treated with chemo-ICI $(n=11)$. Asterisks indicate that patient was not treated with ROS1 TKI. (C) Box and whisker plot revealing median time on each respective treatment. Median time on therapy with range displayed above each treatment category. Chemo-ICl, chemotherapy with immune checkpoint inhibitor; ICl, immune checkpoint inhibitor; ID, identification; PD, progressive disease; TKI, tyrosine kinase inhibitor. 
ICI monotherapy in patients with ROS1-rearranged NSCLCs. This does not mean that providers should select chemo-ICI over ROS1 TKI therapy in treatment-naive patients when the presence of a ROS1 fusion is known. ROS1 TKI remains the therapy of choice in current guidelines on the basis of prospective trials which reveal that ROS1 TKI therapy achieves prolonged overall disease control (median PFS $=19-21 \mathrm{mo}$ ).

Other important considerations are whether patients should be treated with chemo-ICI versus chemotherapy alone or chemotherapy with continued TKI, questions these data were not poised to answer. It is possible that the benefit patients received from chemo-ICI was primarily a benefit from receiving platinum chemotherapy. Although we did not evaluate outcomes on chemotherapy, other studies reported response rates of $45 \%$ to $60 \%$ and a median PFS of 5 to 23 months with various chemotherapy regimens. ${ }^{21-23}$ Furthermore, no prospective data yet support the continuation of ROS1 TKI therapy postprogression with chemotherapy or chemo-ICI. In EGFR-mutant lung cancers, the continuation of gefitinib with chemotherapy after progression on gefitinib did not prolong PFS. ${ }^{47}$ Importantly, the possibility for increased adverse events when immune checkpoint inhibition and TKI therapy are administered in proximity should always be taken into account as this is an established phenomenon in EGFR-mutant ${ }^{48}$ and $A L K$ fusion-positive lung cancers. ${ }^{49}$ In patients previously treated with TKI therapy, the subsequent use of immunotherapyinclusive regimens may have safety implications that have not been prospectively evaluated.

Our study has several notable limitations. This included a sample size that limited our ability to detect meaningful differences in activity by immune biomarker, potential sampling bias from the participating institutions, the lack of defined scanning intervals, and the receipt of immunotherapy at varying points during patients' disease course. From a pathologic perspective, multiple PD-L1 antibodies and NGS panels were used and PD-L1/TMB were not routinely evaluated in treatment-naive samples.

In conclusion, the immunophenotype of ROS1-rearranged NSCLCs is characterized by low or no PD-L1 expression and low TMB in most patients. Although the activity of single-agent immunotherapy was modest, the activity of chemoimmunotherapy was more encouraging.

\section{CRediT Authorship Contribution Statement}

Noura J. Choudhury: Conceptualization, Data curation, Methodology, Writing-original draft, Writing-review and editing.
Jaime L. Schneider: Data curation, Writing-original draft, Writing-review and editing.

Tejas Patil, Andrew Do, Yunan Nie, Jamie E. Chaft, and Christina J. Falcon: Data curation, Writing-review and editing.

Viola W. Zhu: Conceptualization, Data curation, Writing-review and editing.

Debra A. Goldman: Formal analysis, Writing-review and editing.

Soo-Ryum Yang: Data curation, Visualization, Writing-editing and review.

Andrew J. Plodkowski, Subba R. Digumarthy, Natasha Rekhtman, and Maria E. Arcila: Data curation, Resources, Writing-review and editing.

Alexia Iasonos: Formal analysis, Supervision, Writing-review and editing.

Sai-Hong I. Ou: Data curation, Supervision, Writingreview and editing.

Jessica J. Lin and Alexander Drilon: Conceptualization, Data curation, Supervision, Methodology, Writing—original draft, Writing_review and editing.

\section{Acknowledgments}

This work was supported by the National Cancer Institute at the National Institutes of Health P30 CA00874. Dr. Schneider is supported by the National Cancer Institute under grant T32CA071345.

\section{Supplementary Data}

Note: To access the supplementary material accompanying this article, visit the online version of the JTO Clinical and Research Reports at www.jtocrr.org and at https://doi.org/10.1016/j.jtocrr.2021.100187.

\section{References}

1. Soria JC, Ohe Y, Vansteenkiste J, et al. Osimertinib in untreated EGFR-mutated advanced non-small-cell lung cancer. N Engl J Med. 2018;378:113-125.

2. Planchard D, Smit EF, Groen HJM, et al. Dabrafenib plus trametinib in patients with previously untreated BRAFV600E-mutant metastatic non-small-cell lung cancer: an open-label, phase 2 trial. Lancet Oncol. 2017; 18:1307-1316.

3. Paik PK, Felip E, Veillon R, et al. Tepotinib in non-smallcell lung cancer with MET exon 14 skipping mutations. N Engl J Med. 2020;383:931-943.

4. Shaw AT, Bauer TM, de Marinis F, et al. First-line lorlatinib or crizotinib in advanced ALK-positive lung cancer. N Engl J Med. 2020;383:2018-2029.

5. Peters S, Camidge DR, Shaw AT, et al. Alectinib versus crizotinib in untreated ALK-positive non-small-cell lung cancer. N Engl J Med. 2017;377:829-838.

6. Drilon A, Jenkins C, lyer S, Schoenfeld A, Keddy C, Davare MA. ROS1-dependent cancers - biology, diagnostics and therapeutics. Nat Rev Clin Oncol. 2021;18:35-55. 
7. Drilon A, Siena S, Dziadziuszko R, et al. Entrectinib in ROS1 fusion-positive non-small-cell lung cancer: integrated analysis of three phase 1-2 trials. Lancet Oncol. 2020;21:261-270.

8. Drilon A, Oxnard GR, Tan DSW, et al. Efficacy of selpercatinib in RET fusion-positive non-small-cell lung cancer. N Engl J Med. 2020;383:813-824.

9. Drilon A, Laetsch TW, Kummar S, et al. Efficacy of larotrectinib in TRK fusion-positive cancers in adults and children. N Engl J Med. 2018;378:731-739.

10. Jordan EJ, Kim HR, Arcila ME, et al. Prospective comprehensive molecular characterization of lung adenocarcinomas for efficient patient matching to approved and emerging therapies. Cancer Discov. 2017;7:596-609.

11. Bergethon K, Shaw AT, Ou SH, et al. ROS1 rearrangements define a unique molecular class of lung cancers. J Clin Oncol. 2012;30:863-870.

12. Drilon A, Siena S, Ou SI, et al. Safety and antitumor activity of the multitargeted pan-TRK, ROS1, and ALK inhibitor entrectinib: combined results from two phase I trials (ALKA-372-001 and STARTRK-1). Cancer Discov. 2017;7:400-409.

13. Shaw AT, Ou SHI, Bang YJ, et al. Crizotinib in ROS1rearranged non-small-cell lung cancer. N Engl J Med. 2014;371:1963-1971.

14. Moro-Sibilot D, Cozic N, Pérol M, et al. Crizotinib in CMET- or ROS1-positive NSCLC: results of the AcSé phase II trial. Ann Oncol. 2019;30:1985-1991.

15. Landi L, Chiari R, Tiseo M, et al. Crizotinib in METderegulated or ROS1-rearranged pretreated non-small cell lung cancer (METROS): a phase II, prospective, multicenter, two-arms trial. Clin Cancer Res. 2019;25:7312-7319.

16. Shaw AT, Solomon BJ, Chiari R, et al. Lorlatinib in advanced ROS1-positive non-small-cell lung cancer: a multicentre, open-label, single-arm, phase 1-2 trial. Lancet Oncol. 2019;20:1691-1701.

17. Cho BC, Doebele RC, Lin J, et al. Phase 1/2 TRIDENT-1 study of repotrectinib in patients with $\mathrm{ROS} 1+$ or NTRK+ advanced solid tumors. J Thorac Oncol. 2021;16(suppl):S174-S175.

18. Papadopoulos KP, Borazanci E, Shaw AT, et al. U.S. phase I first-in-human study of taletrectinib (DS-6051b/AB106), a ROS1/TRK inhibitor, in patients with advanced solid tumors. Clin Cancer Res. 2020;26:4785-4794.

19. Ou S-HI, Fujiwara Y, Shaw AT, et al. Efficacy of taletrectinib (AB-106/DS-6051b) in ROS1+ NSCLC: an updated pooled analysis of U.S. and Japan phase 1 studies. JTO Clin Res Rep. 2021;2:100108.

20. National Comprehensive Cancer Network. NCCN clinical practice guidelines in oncology. https: //www.nccn.org/ login?ReturnURL=https: / /www.nccn.org/professionals / physician_gls/pdf/nscl.pdf. Accessed February 28, 2020.

21. Park S, Ahn BC, Lim SW, et al. Characteristics and outcome of ROS1-positive non-small cell lung cancer patients in routine clinical practice. J Thorac Oncol. 2018;13:1373-1382.

22. Kim HR, Lim SM, Kim HJ, et al. The frequency and impact of ROS1 rearrangement on clinical outcomes in never smokers with lung adenocarcinoma. Ann Oncol. 2013;24:2364-2370.
23. Chen YF, Hsieh MS, Wu SG, et al. Efficacy of pemetrexed-based chemotherapy in patients with ROS1 fusion-positive lung adenocarcinoma compared with in patients harboring other driver mutations in east Asian populations. J Thorac Oncol. 2016;11:11401152.

24. Rangachari D, VanderLaan PA, Shea M, et al. Correlation between classic driver oncogene mutations in EGFR, ALK, or ROS1 and 22C3-PD-L1 $\geq 50 \%$ expression in lung adenocarcinoma. J Thorac Oncol. 2017;12:878-883.

25. Jiang L, Su X, Zhang T, et al. PD-L1 expression and its relationship with oncogenic drivers in non-small cell lung cancer (NSCLC). Oncotarget. 2017;8:26845-26857.

26. Mazieres J, Drilon A, Lusque A, et al. Immune checkpoint inhibitors for patients with advanced lung cancer and oncogenic driver alterations: results from the IMMUNOTARGET registry. Ann Oncol. 2019;30:1321-1328.

27. Jain A, Fujioka N, Patel M. Immune checkpoint inhibitors in ROS1-rearranged non-small cell lung cancer: a report of two cases. J Thorac Oncol. 2019;14:e165-e167.

28. Mok TSK, Wu YL, Kudaba I, et al. Pembrolizumab versus chemotherapy for previously untreated, PD-L1expressing, locally advanced or metastatic non-small-cell lung cancer (KEYNOTE-042): a randomised, open-label, controlled, phase 3 trial. Lancet. 2019;393:1819-1830.

29. Reck M, Rodriguez-Abreu D, Robinson AG, et al. Pembrolizumab versus chemotherapy for PD-L1-positive non-small-cell lung cancer. N Engl J Med. 2016;375: 1823-1833.

30. Hellmann MD, Ciuleanu TE, Pluzanski A, et al. Nivolumab plus ipilimumab in lung cancer with a high tumor mutational burden. N Engl J Med. 2018;378:2093-2104.

31. Marabelle A, Fakih M, Lopez J, et al. Association of tumour mutational burden with outcomes in patients with advanced solid tumours treated with pembrolizumab: prospective biomarker analysis of the multicohort, open-label, phase 2 KEYNOTE-158 study. Lancet Oncol. 2020;21:1353-1365.

32. Herbst RS, Baas P, Kim DW, et al. Pembrolizumab versus docetaxel for previously treated, PD-L1-positive, advanced non-small-cell lung cancer (KEYNOTE-010): a randomised controlled trial. Lancet. 2016;387:1540-1550.

33. Cheng DT, Mitchell TN, Zehir A, et al. Memorial Sloan Kettering-integrated mutation profiling of actionable cancer targets (MSK-IMPACT): a hybridization capturebased next-generation sequencing clinical assay for solid tumor molecular oncology. J Mol Diagn. 2015; 17:251-264.

34. Cerami E, Gao J, Dogrusoz U, et al. The cBio cancer genomics portal: an open platform for exploring multidimensional cancer genomics data. Cancer Discov. 2012;2:401-404.

35. Skoulidis F, Arbour KC, Hellmann MD, et al. Association of STK11/LKB1 genomic alterations with lack of benefit from the addition of pembrolizumab to platinum doublet chemotherapy in non-squamous non-small cell lung cancer. J Clin Oncol. 2019;37(suppl 15):102-102.

36. Liu J, Zhou H, Zhang $\mathrm{Y}$, et al. PBRM1 mutation and preliminary response to immune checkpoint blockade treatment in NSCLC. Ann Oncol. 2019;30(suppl 9):ix107ix114. 
37. Papillon-Cavanagh S, Doshi P, Dobrin R, Szustakowski J, Walsh AM. STK11 and KEAP1 mutations as prognostic biomarkers in an observational real-world lung adenocarcinoma cohort. ESMO Open. 2020;5:e000706.

38. Offin M, Rizvi H, Tenet M, et al. Tumor mutation burden and efficacy of EGFR-tyrosine kinase inhibitors in patients with EGFR-mutant lung cancers. Clin Cancer Res. 2019;25:1063-1069.

39. Davis AA, Chae YK, Agte S, et al. Association of tumor mutational burden with smoking and mutation status in non-small cell lung cancer (NSCLC). J Clin Oncol. 2017;35(suppl 7):24-24.

40. Offin M, Guo R, Wu SL, et al. Immunophenotype and response to immunotherapy of RET-rearranged lung cancers. JCO Precis Oncol. 2019;3:PO.18.00386.

41. Hastings K, Yu HA, Wei W, et al. EGFR mutation subtypes and response to immune checkpoint blockade treatment in non-small-cell lung cancer. Ann Oncol. 2019;30:13111320.

42. Lisberg A, Cummings A, Goldman JW, et al. A phase II study of pembrolizumab in EGFR-mutant, PD-L1+, tyrosine kinase inhibitor naïve patients with advanced NSCLC. J Thorac Oncol. 2018;13:1138-1145.

43. Gainor JF, Shaw AT, Sequist LV, et al. EGFR mutations and ALK rearrangements are associated with low response rates to PD-1 pathway blockade in non-small cell lung cancer: a retrospective analysis. Clin Cancer Res. 2016;22:4585-4593.
44. Dudnik E, Peled N, Nechushtan H, et al. BRAF mutant lung cancer: programmed death ligand 1 expression. Tumor mutational burden, microsatellite instability status, and response to immune check point inhibitors. J Thorac Oncol. 2018;13:1128-1137.

45. Sabari JK, Leonardi GC, Shu CA, et al. PD-L1 expression, tumor mutational burden, and response to immunotherapy in patients with MET exon 14 altered lung cancers. Ann Oncol. 2018;29:2085-2091.

46. Gandhi L, Rodriguez-Abreu D, Gadgeel S, et al. Pembrolizumab plus chemotherapy in metastatic nonsmall-cell lung cancer. N Engl J Med. 2018;378: 2078-2092.

47. Soria JC, Wu YL, Nakagawa K, et al. Gefitinib plus chemotherapy versus placebo plus chemotherapy in EGFR-mutation-positive non-small-cell lung cancer after progression on first-line gefitinib (IMPRESS): a phase 3 randomised trial. Lancet Oncol. 2015;16: 990-998.

48. Schoenfeld AJ, Arbour KC, Rizvi H, et al. Severe immune-related adverse events are common with sequential PD-(L)1 blockade and osimertinib. Ann Oncol. 2019;30:839-844.

49. Lin JJ, Chin E, Yeap BY, et al. Increased hepatotoxicity associated with sequential immune checkpoint inhibitor and crizotinib therapy in patients with non-small cell lung cancer. J Thorac Oncol. 2019;14: 135-140. 